\title{
Reduced Order System Identification for UAVs
}

\section{P-D Jameson and A K Cooke}

\author{
Department of Aerospace Engineering \\ Cranfield University \\ Bedfordshire \\ MK43 0AL \\ United Kingdom \\ pierre_jameson@msn.com, a.cooke@cranfield.ac.uk
}

\begin{tabular}{|c|c|c|}
\hline Abstract & $\begin{array}{l}b \\
b(k)\end{array}$ & $\begin{array}{l}\text { Wing span, }(\mathrm{m}) \\
\text { Fourier sine series coefficients }\end{array}$ \\
\hline Reduced order models representing the dynamic be- & $g$ & Signal time history \\
\hline haviour of symmetric aircraft are well known and & $h$ & Altitude, (ft) \\
\hline can be easily derived from the standard equations & $k$ & Filter cutoff frequency, $(\mathrm{Hz})$ \\
\hline of motion. In flight testing, accurate measurements & $m_{\{.\}}$ & Concise pitching moment derivative \\
\hline of the dependent variables which describe the lin- & $n$ & Number of discrete points \\
\hline earised reduced order models for a particular flight & $n_{\{.\}}$ & Concise yawing moment derivative \\
\hline condition are vital for successful system identifica- & $p$ & Roll rate, (deg/s) \\
\hline tion. However, not all the desired measurements & $q$ & Pitch rate, $(\mathrm{deg} / \mathrm{s})$ \\
\hline such as the rate of change in vertical velocity $(\dot{W})$ & $r$ & Yaw rate, $(\mathrm{deg} / \mathrm{s})$ \\
\hline can be accurately measured in practice. In order to & $s$ & Standard error \\
\hline determine such variables two possible solutions ex- & $u$ & Input vector \\
\hline ist: reconstruction or differentiation. This paper ad- & $x$ & State vector \\
\hline dresses the effect of both methods on the reliability & $y$ & Observation vector \\
\hline of the parameter estimates. The methods are used in & $y_{\{.\}}$ & Concise lateral force derivative \\
\hline the estimation of the aerodynamic derivatives for the & $z$ & Measurement vector \\
\hline Aerosonde UAV from a recreated flight test scenario & $z_{\{.\}}$ & Concise normal force derivative \\
\hline in Simulink@). Subsequently, the methods are then & A & State matrix \\
\hline applied and compared using real data obtained from & B & Input matrix \\
\hline flight tests of the Cranfield University Jetstream-31 & $\mathrm{E}$ & Expectation operator \\
\hline (G-NFLA) research aircraft. & I & Identity matrix \\
\hline Keywords: Parameter Estimation, System & $\mathbf{P}$ & Parameter covariance matrix \\
\hline Identification & $U$ & Total axial velocity, (m/s) \\
\hline & $V$ & Total lateral velocity, (m/s) \\
\hline & $V_{T A S}$ & True Airspeed, (kts) \\
\hline Nomenclature & $V_{0}$ & Trim true airspeed, $(\mathrm{m} / \mathrm{s})$ \\
\hline & $W$ & Total normal velocity, (m/s) \\
\hline Null matrix & $\mathbf{X}$ & Matrix of regressors \\
\hline Acceleration, $\left(\mathrm{m} / \mathrm{s}^{2}\right)$ & & \\
\hline
\end{tabular}




\section{Greek letters}

$\begin{array}{ll}\alpha & \text { Angle of attack, (deg) } \\ \beta & \text { Angle of sideslip, (deg) } \\ \Delta t & \text { Pulse width, (sec) } \\ \epsilon & \text { Equation error } \\ \zeta & \text { Rudder deflection, (deg) } \\ & \text { Damping coefficient } \\ \eta & \text { Elevator deflection, (deg) } \\ \theta & \text { Parameter estimate } \\ \sigma & \text { Variance } \\ \phi(k) & \text { Wiener filter weighting } \\ \omega & \text { Frequency of oscillation, }(\mathrm{rad} / \mathrm{s}) \\ \omega_{d r} & \text { DR natural frequency, }(\mathrm{rad} / \mathrm{s}) \\ \omega_{s p} & \text { SPPO natural frequency, }(\mathrm{rad} / \mathrm{s}) \\ \omega_{n} & \text { Natural frequency, }(\mathrm{rad} / \mathrm{s})\end{array}$

\section{Superscripts}

$\begin{array}{ll}\hat{T} & \text { Transpose } \\ . & \text { Estimate } \\ \circ & \text { Derivative with respect to time } \\ & \text { Degrees }\end{array}$

\section{Subscripts}

$\begin{array}{ll}0 & \text { Initial condition } \\ \text { bias } & \text { Bias in measured variable } \\ s & \text { Smoothed variable } \\ \text { trim } & \text { Trim condition } \\ \text { off } & \text { Sensor offset from c.g in Cartesian } \\ & \text { coordinates, }(\mathrm{m}) \\ x, y, z & \text { Respective axes }\end{array}$

\section{Abbreviations}

$\begin{array}{ll}\text { c.g } & \text { Centre of gravity } \\ \text { DCC } & \text { Data compatibility check } \\ \text { Dif } & \text { Differentiated } \\ \text { DOF } & \text { Degree of freedom } \\ \text { DR } & \text { Dutch roll } \\ \text { EE } & \text { Equation error } \\ \text { IRS } & \text { Inertial reference system } \\ \text { MAC } & \text { Mean aerodynamic chord } \\ \text { MTOW } & \text { Maximum take-off weight } \\ \text { OLS } & \text { Ordinary least squares }\end{array}$

$\begin{array}{ll}\text { PE } & \text { Parameter estimation } \\ \text { PID } & \text { Parameter identification } \\ \text { Rec } & \text { Reconstructed } \\ \text { Sim } & \text { Simulated } \\ \text { SLF } & \text { Steady level flight } \\ \text { S/N } & \text { Signal to noise ratio } \\ \text { SPPO } & \text { Short period pitching oscillation } \\ \text { SysID } & \text { System identification }\end{array}$

\section{Introduction}

Examples of applying system identification to flight test data of manned aircraft have been widely reported, [1] and [2]. Due to their sometimes unconventional and complex shapes, rapid and effective estimation for UAVs is essential as it may be impossible to obtain suitable data for a UAVs stability characteristics necessary for it to fly without having first flown. For such reasons recent work at Cranfield has concentrated on investigating suitable system identification techniques for use with UAVs [3]. It is important to underline the benefits that could be gained from developing system identification use with UAVs since accurate knowledge of their stability and control characteristics is vital for autonomous flight.

Applying System identification (SysID) techniques to the flight test data enables us to maximise the amount of useful information gathered within a given time period. However, it must be stated that measurements easily achieved during wind tunnel tests can prove to be cumbersome in-flight. One such example is the ability to gather high quality data without noise due to atmospheric turbulence.

Furthermore, wind tunnels provide a safer means for testing unstable areas of the flight envelope such as high angle-of-attack $(\alpha)$ or side-slip $(\beta)$. SysID can also play a role in high- $\alpha$ or $-\beta$ flight if it is possible to gather sufficient data for analysis during a short excursion into this region of the flight envelope. Usually, flight testing for SysID purposes involves performing small perturbations about a steady and level flight condition known as trim.

Focusing on smaller UAVs $(b<4 m)$ an inherent problem is the difficulty with which reliable air data can be obtained. Without air data and using current 
practice of differentiation, the required variables for SysID such as $\dot{W}$ and $\dot{V}$ cannot be obtained. However, one of the aims of this paper is to suggest a solution to this problem whereby $\dot{W}$ and $\dot{V}$ can be reconstructed using inertial measurements. In addition, identification using reduced order models helps to reduce the computational complexity which will be of benefit for online/onboard SysID envisaged in future work. Therefore, the research presented is separated into two parts: firstly, an investigation using a Simulink model of the Aerosonde UAV (figure 1) is performed. Secondly, to provide a real-world example, data recorded from the Cranfield University BAe Jetstream-31 aircraft (G-NFLA) is used to verify the simulation results.

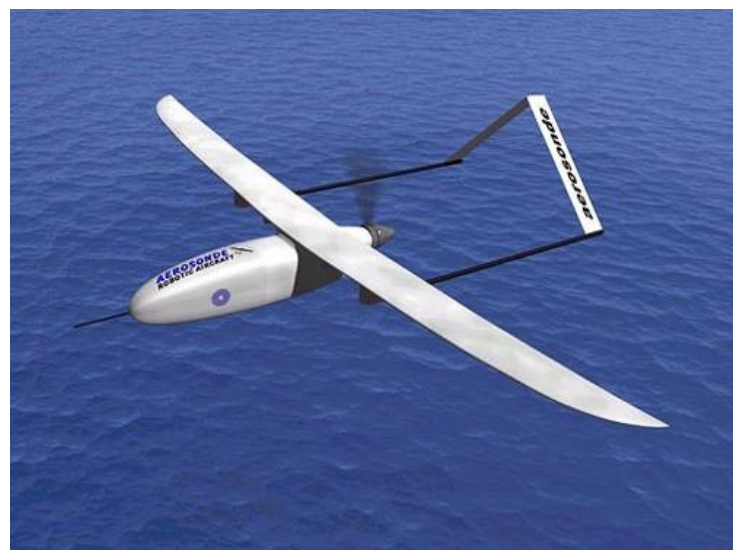

Figure 1: The Aerosonde UAV [4].

This paper is divided into five further sections: firstly, the methods used for analysis are presented, secondly the results for a simulated Aerosonde UAV, as well as the results from real flight data are presented. These results are then discussed, and finally, conclusions and future work are outlined.

\section{Method}

\subsection{System Identification Explained}

In order to aid the reader, pertinent abbreviations specific to this field and used herein are brought together in the nomenclature. Before discussing the mathematical principles behind the identification techniques it is useful to establish a definition of aircraft system identification. In the context of aerospace, Parameter estimation (PE)/Parameter identification (PID) and System identification (SysID) are frequently used interchangeably and are hence considered synonymous. However, it is important to note that in other fields of identification PID describes a simplified case of SysID; where the system's model is postulated a priori. As early as 1962 Zadeh [5] provided us with a succinct description of system identification: "Identification is the determination, on the basis of observation of input and output, of a system within a specified class of systems to which the system under test is equivalent". More recently Hamel and Jategaonkar [2] presented a simpler definition: "Given the system responses, what is the model?".

Due to the complexity of the methods employed in SysID, the above definitions are a useful means of keeping our final objective in view.

\subsection{Aircraft System Identification}

A summary of the full aircraft SysID procedure can be found in [6]. The SysID process (figure 2) stems from the a priori knowledge of the aircraft and can be seen as a further five distinct steps: experiment design, data compatibility check, model structure determination, parameter estimation and model validation.

In order to be able to perform $\mathrm{PE}$ the aircraft needs to be suitably excited so that its underlying dynamics "show themselves". Subsequently, the role of the Data Compatibility Check (DCC) is to provide a means of pre-processing/checking the data before it is used by the PE algorithms; the data is inspected for gross errors such as incorrect signs, data drop-outs and extensive noise [7]. Furthermore, during DCC, reconstruction of measured variables is used to verify the kinematic consistency of the recorded data such as comparing the estimated state against the measured state.

As the data recorded for both the Aerosonde and Jetstream-31 are known to be compatible the DCC step was bypassed. Furthermore, when a postulated 


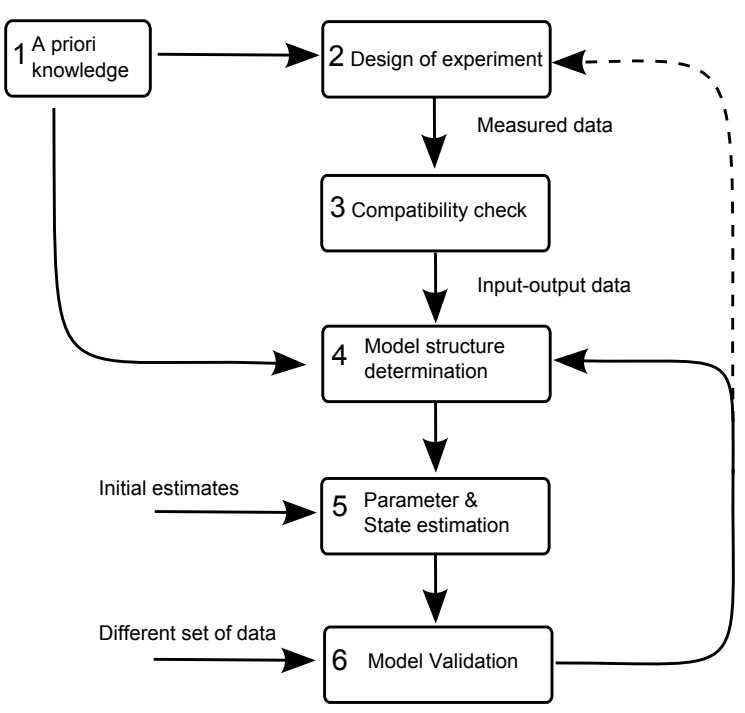

Figure 2: An overview of the SysID process, [7].

aircraft model structure (determined from a priori knowledge [8]) is used, the model structure determination step is made redundant, and as a result, the current analysis proceeded directly from the experimental measurements to the PE step.

\section{$2.3 \quad$ Equation Error}

When performing PE, a key decision needs to be made regarding which identification technique should be used. Such a decision needs to be made with the context of the application in mind, and for this work, the aim was to provide timely results, to facilitate real-time implementation. Therefore, it was decided to select the Equation Error (EE) formulation in the time domain for parameter estimation. Simply, EE is a technique which estimates the aircraft parameters with a linear regression using the Ordinary least squares (OLS) technique [6]. In order to apply this technique the equations of motion are assumed to be in the form:

$$
y(t)=\theta_{0}+\theta_{1} X_{1}(t)+\theta_{2} X_{2}(t) \ldots+\theta_{n} X_{n}(t)
$$

where $y$ is the dependent variable, $X_{1}, X_{2}, \ldots X_{n}$ are the $n$ unique regressors and $\theta_{0}, \theta_{1}, \theta_{2}, \ldots \theta_{n}$ are the $n$ unknown parameters which need to be determined. The dependent variables and unique regressors which are the topic of the current paper are assumed to be available either by direct measurement or calculation. The mathematical expression now follows with the measurement equation:

$$
\begin{aligned}
z(i) & =y(i)+\epsilon(i) \\
& =\theta_{0}+\sum_{i=0}^{N} \theta_{j} X_{j}(i)+\epsilon(i), \quad i=1,2 \ldots N
\end{aligned}
$$

where $z(i)$ is the measured value of the dependent variable $y(i)$ at the $i^{t h}$ data point, $\epsilon(i)$ accounts for the random error corrupting the true value of $y(i)$ and $N$ represents the number of discrete data points taken for the analysis. Writing equation (2) in matrix form yields:

$$
z=\mathbf{X} \boldsymbol{\theta}+\boldsymbol{\epsilon}
$$

Being able to formulate the problem in matrix form facilitates computational manipulation of the large data sets collected during a test. It must be noted that the members of the EE matrix, $\boldsymbol{\epsilon}$ are assumed to be uncorrelated with the regressors. Therefore, the values of $\boldsymbol{\epsilon}$ are represented as white noise with zero mean and variance $\sigma^{2}, E[\boldsymbol{\epsilon}]=\mathbf{0}$ and $E\left[\boldsymbol{\epsilon} \boldsymbol{\epsilon}^{T}\right]=\sigma^{2} \mathbf{I}$; note that $E$ is the expectation operator and $\mathbf{I}$ represents the identity matrix.

Having outlined the necessary equations for equation (3), the least squares technique can now be used to determine the unknown parameters $\boldsymbol{\theta}$ by minimising the cost function for the sum of the squares of the equation error $\boldsymbol{\epsilon}$, formulated as:

$$
\begin{aligned}
J(\theta) & =\frac{1}{2} \sum_{i=1}^{N} \boldsymbol{\epsilon}^{2}(i)=\frac{1}{2} \boldsymbol{\epsilon}^{T} \boldsymbol{\epsilon} \\
& =\frac{1}{2}(\boldsymbol{z}-\mathbf{X} \boldsymbol{\theta})^{T}(\boldsymbol{z}-\mathbf{X} \boldsymbol{\theta})
\end{aligned}
$$

The minimum is then found by differentiating equation (4) with respect to $\boldsymbol{\theta}$ and setting the equation equal to zero:

$$
\frac{\partial J(\boldsymbol{\theta})}{\partial \boldsymbol{\theta}}=-\boldsymbol{z}^{T} \mathbf{X}+\boldsymbol{\theta}^{T} \mathbf{X}^{T} \mathbf{X}=0
$$


Hence the normal (orthogonal) equation can be obtained by taking the transpose and re-arranging equation (5):

$$
\mathbf{X}^{T} \mathbf{X} \hat{\boldsymbol{\theta}}=\mathbf{X}^{T} \boldsymbol{z}
$$

Here $\mathbf{X}^{T} \mathbf{X}$ is known as the information matrix because it provides a measure of the information content in the data. Now assuming that the information matrix can be inverted, the normal equation can be re-arranged as follows:

$$
\hat{\boldsymbol{\theta}}=\left(\mathbf{X}^{T} \mathbf{X}\right)^{-1} \mathbf{X}^{T} \boldsymbol{z}
$$

where, $\hat{\boldsymbol{\theta}}$ is the least squares estimate of the true unknown parameter vector $\boldsymbol{\theta}$. The following properties can be outlined for the parameter estimates:

1. They are unbiased, $\mathrm{E}[\hat{\boldsymbol{\theta}}]=\boldsymbol{\theta}$

2. They are consistent, so that as the number of data points $N$ increases the least squares estimates converge on the true parameter estimates

3. They are an efficient estimate, such that the parameter covariance is given by: $\mathbf{P}=\sigma^{2}\left[\mathbf{X}^{T} \mathbf{X}\right]$

Having stated the properties above it should be mentioned that for the purposes of the OLS method two important assumptions have been made: 1) the regressors, $X_{n}$ in equation (1) are assumed to be known without error and 2) the noise is assumed to be white and Gaussian. In the case of the simulated Aerosonde UAV these are true, however, in the case of real aircraft data these are both clearly violated. For real data the estimated parameters will be biased and inefficient; the extent to which this occurs is dependent on the noise level, nevertheless, these can be mitigated through the choice of instrumentation and careful data handling [9].

\subsection{Reduced Order Models}

Using the equations of motion the dynamic behaviour of an aircraft can be accurately described. When performing PE a priori knowledge is crucial to choosing a suitable model to represent the dynamic mode under investigation. In this paper the longitudinal reduced order Short period pitching oscillation (SPPO, equation (8)) and the lateral Dutch roll mode (DR, equation (9)) are used for analysis.

Selection of reduced order models assumes the decoupling of the longitudinal and lateral dynamics; this simplifies PE and is based on the assumption that the air vehicle is symmetric. In the asymmetric case e.g. due to aircraft damage or a change in external stores, the full order equations will be required in order to account for the cross term derivatives due to the potential for cross-coupling between the different dynamic modes [10]. Nevertheless, reduced order models enable us to gain an overview of the characteristics before proceeding with the more complex full-order coupled models.

It is important to understand the effect of determining the state variables on the outcome of the aerodynamic derivatives as these form the basis of the dynamic models capable of describing the behaviour of the aircraft for handling quality and control law design. Converting the aerodynamic derivatives into dimensional form enables us to gain a quantitative understanding of the airframe design from the recorded data. The following body axes models are taken from [8]; the SPPO model is:

$$
\left[\begin{array}{c}
\dot{\boldsymbol{W}} \\
\dot{\boldsymbol{q}}
\end{array}\right]=\left[\begin{array}{cc}
z_{w} & z_{q} \\
m_{w} & m_{q}
\end{array}\right]\left[\begin{array}{c}
\boldsymbol{W} \\
\boldsymbol{q}
\end{array}\right]+\left[\begin{array}{c}
z_{\eta} \\
m_{\eta}
\end{array}\right][\boldsymbol{\eta}]
$$

and the Dutch roll model is:

$$
\left[\begin{array}{c}
\dot{\boldsymbol{V}} \\
\dot{\boldsymbol{r}}
\end{array}\right]=\left[\begin{array}{ll}
y_{v} & y_{r} \\
n_{v} & n_{r}
\end{array}\right]\left[\begin{array}{c}
\boldsymbol{V} \\
\boldsymbol{r}
\end{array}\right]+\left[\begin{array}{c}
y_{\zeta} \\
n_{\zeta}
\end{array}\right][\boldsymbol{\zeta}]
$$

Here it must be noted that the above stability and control derivatives are in concise form, and therefore require manipulation in order to relate them to the physical system.

\subsection{Reconstruction}

The variables that cannot be measured directly, so coined as "unmeasurables", pose a problem that needs to be solved with minimal additional error. During the course of a test, data is recorded from onboard instrumentation such as accelerometers $\left(a_{x}, a_{y}, a_{z}\right)$, angular rate gyros $(p, q, r)$, and air data measurements such as $\alpha$-vanes to name a few. 
However, the ability to measure some variables with current instruments still proves to be difficult, furthermore, in the case of a UAV the cost of such instrumentation and possible weight limitations may further reduce the number of variables that can be measured.

Already in the case of the angular accelerations $\dot{p}, \dot{q}$, and $\dot{r}$ instrumentation is not used as differentiation of the direct measurements $p, q$, and $r$ is suitable and represents current practice [11],[12].

$\dot{W}$ and $\dot{q}$ are the two unmeasuarables for the reduced order SPPO mode (see section 2.4). Therefore in the SPPO analysis $\dot{q}$ will be determined from differentiation for both the Aerosonde and Jetstream investigations. Starting from a trim condition, and using the equations of motion for a rigid body, [8] we obtain:

$$
\begin{gathered}
a_{z}=\dot{W}-q U+p V+x_{\mathrm{off}}(p r-\dot{q}) \\
+y_{\mathrm{off}}(q r+\dot{p})-z_{\mathrm{off}}\left(p^{2}+q^{2}\right)
\end{gathered}
$$

and making the assumption that the motion is decoupled, the reconstruction equation for $\dot{W}$ yields:

$$
\dot{W}=a_{z}+q U+x_{\text {off }} \dot{q}+z_{\text {off }} q^{2}
$$

In the Dutch roll analysis the unmeasureables are $\dot{V}, \dot{p}$ and $\dot{r}$ where differentiation of yaw rate $r$ and roll rate $p$ yields $\dot{r}$ and $\dot{p}$ respectively. Again assuming that the motion is decoupled and starting from a trim condition:

$$
\begin{gathered}
a_{y}=\dot{V}-p W+r U+x_{\mathrm{off}}(p q+\dot{r}) \\
-y_{\mathrm{off}}\left(p^{2}+r^{2}\right)+z_{\mathrm{off}}(q r-\dot{p})
\end{gathered}
$$

$\dot{V}$ is obtained by reconstruction from:

$$
\dot{V}=a_{y}-r U-x_{\mathrm{off}} \dot{r}+y_{\mathrm{off}}\left(p^{2}+r^{2}\right)+z_{\mathrm{off}} \dot{p}
$$

However, in the case of the Aerosonde simulation the measured rates are about the c.g and therefore $x_{\text {off }}$ $=y_{\mathrm{off}}=z_{\mathrm{off}}=0$. Equations (11) and (13) further simplify to:

$$
\begin{aligned}
\dot{W} & =a_{z}+q U \\
\dot{V} & =a_{y}-r U
\end{aligned}
$$

Furthermore, for the Aerosonde simulation the recorded data by definition will not include measurement errors, therefore performing either reconstruction or differentiation will not enable us to draw any firm conclusions regarding their performance. However, in comparison to the atmospheric flight test the simulation environment enables us to accurately linearise our aircraft model containing the aerodynamic derivatives hence providing the true values for analysis. A further advantage of using a simulation is that additive noise can be applied specifically to the $U$, $V, W, p, q, r, a_{y}$, and $a_{z}$ channels without affecting any of the other channels enabling us to analyse the effects of reconstruction or differentiation on the resulting estimated aerodynamic derivatives.

\subsection{Differentiation}

As one of the aims of this paper is to compare the difference between results for when the state variables are obtained from either reconstruction or differentiation, an explanation of the differentiation technique used to obtain $\dot{W}$ and $\dot{V}$ for comparison now follows. Using the small angles approximation vertical and lateral velocities can be calculated directly from the air data measurements using:

$$
\begin{gathered}
W=V_{0} \sin (\alpha) \cong V_{0} \times \alpha \\
V=V_{0} \sin (\beta) \cong V_{0} \times \beta
\end{gathered}
$$

The method for differentiation is taken from [9], which incorporates the differentiation as part of a global Fourier smoothing algorithm. Smoothing before differentiation is done to minimise the noise present in the data. The cutoff frequency for the data needs to be chosen in order to remove structural vibrations and other high frequency noise from the measured signals. Currently this is performed using a visual plot of the frequency data, and therefore a Wiener filter is included to account for some of the error due to the manual cutoff frequency selection. In future work for online use this choice will need to be replaced by either hard coding a pre-selected cutoff value or through additional data metrics, as suggested by [13].

The Fourier transform assumes that the time history $g(i)$ is periodic i.e. $g(-N)=g(1)=g(N)=0$, 
so it is necessary to remove the start and end points of the data by subtracting a linear trend from the data before reflecting the data about its origin. Subsequently, the vector of data points with the end points removed is an odd function of time that can be approximated by a Fourier sine series:

$$
\begin{aligned}
\hat{g}_{s}(i) & =\sum_{k=1}^{k_{\max }} \phi(k) b(k) \sin \left[k \pi\left(\frac{i-1}{N-1}\right)\right] \\
i & =1,2, \ldots, N
\end{aligned}
$$

and $k_{\max }$ is the cutoff frequency chosen from the plot of the Fourier sine series, $\phi(k)$ is the Wiener filter weighting at each frequency and $b(k)$ are the Fourier sine series coefficients. The smoothed values are then obtained by adding the linear trend removed from the data:

$$
\begin{aligned}
z_{s}(i) & =\hat{g}_{s}(i)+z(1)+(i-1)\left[\frac{z(N)-z(1)}{N-1}\right] \\
i & =1,2, \ldots, N
\end{aligned}
$$

Following on from this result the derivative of the smoothed signal with respect to time can be obtained by differentiating the sine series and the linear trend that was removed from the smoothed values:

$$
\begin{aligned}
\dot{z}_{s} & =\left[\frac{z(N)-z(1)}{N-1}\right] \\
& +\sum_{k=1}^{k_{\max }} \phi(k) b(k)\left(\frac{k \pi}{N-1}\right) \cos \left[k \pi\left(\frac{i-1}{N-1}\right)\right] \\
i & =1,2, \ldots, N
\end{aligned}
$$

Note that as $z(1)$ and $z(N)$ are omitted from the global smoothing in cases of severe noisy data this could lead to significant errors. In such cases a local smoothing technique with the same cutoff frequency can be used on the end points before then proceeding with the global smoothing and differentiation method $[9]$.

\subsection{Excitation Input}

When performing PE the aircraft is excited about a steady, wings level trim condition. To be able to apply the Small Disturbance Theory, [14] excitation manoeuvres should not cause the aircraft to deviate excessively from the trim condition. Using a multistep style input such as a doublet or 3-2-1-1 pulse train (jocularly known as the "poor man's frequency sweep") helps perturb the aircraft without departure from trim. Subsequently, many researchers have looked at developing suitable techniques for such purposes [9], [11], and [15]. The main criterion for choosing an input is whether it sufficiently excites the frequency band of interest. Consequently, the doublet input pulse width $\Delta$ t needs to be designed to sufficiently target a bandwidth close to the natural frequency $\omega_{n}$. The following equation is suggested by [11]:

$$
\Delta t=\frac{2.3}{\omega_{n}}
$$

Applying equation (21) necessitates knowing the natural frequency a priori, in the simulated case this is straight forward, however, in practice a good initial estimate can be based on empirical data or wind tunnel measurements and then updated as required. The Aerosonde pulse widths were found to be:

\begin{tabular}{lcc}
\hline Mode & Frequency $(\mathrm{rad} / \mathrm{s})$ & $\Delta \mathrm{t}(\mathrm{s})$ \\
\hline $\mathrm{SPPO}$ & $\omega_{s p} \approx 11.4$ & 0.2018 \\
$\mathrm{DR}$ & $\omega_{d r} \approx 5.98$ & 0.3846 \\
\hline
\end{tabular}

Table 1: Aerosonde doublet pulse widths

From previous experience the inputs used for the Aerosonde were chosen to be $2^{\circ}$ elevator and rudder doublets with the respective pulse widths outlined in table 1. In the case of the Jetstream, the available data for the SPPO mode was excited by an elevator impulse.

When performing manoeuvres for SysID it is important to make sure that the excitation is about a Steady level flight (SLF) trim condition. Therefore, considering the longitudinal SPPO mode, this requires the pilot to select the correct pitch attitude using a small constant elevator deflection $\left(\eta_{\text {trim }}\right)$. In the case of the Aerosonde model a positive $2^{\circ}$ elevator doublet was used to target the natural frequency. In the case of the Jetstream, the flight data available was 
taken from demonstration flights of the SPPO mode where the excitation input consisted of two concatenated elevator impulses performed by the pilot, one positive and one negative.

In either case the trim condition needs to be held constant before applying the excitation. Subsequently, the perturbation values are determined by removing the recorded trim condition from the flight data. This is performed by subtracting the average of each measured variable during the period before the excitation is applied.

\section{Simulated Example}

The Aerosonde UAV is a small UAV $(b=2.9 \mathrm{~m})$ designed primarily for weather-resonnaissance and remote-sensing missions. It has a twin tail boom with a rear mounted pusher propeller configuration with a speed range of 40 - 60kts and a MTOW of $15 \mathrm{~kg}$ [16].

The Aerosonde UAV was chosen as an in-house Simulink model was readily available. By definition the outputs from the simulation will not have any of the stochastic noise effects such as those due to atmospheric disturbance, [17] as well as those due to instrument bias and measurement noise encountered during real flight testing. Furthermore, the ability to use the Linmod function within Matlab enables us to recover the full order ( 6 degrees of freedom) linearised state space equations for the trimmed model which can then be used for comparison with the estimated values.

\subsection{Approach}

In order to investigate the effects of noise on the parameter estimates clean data (direct from the simulation) and noisy data (data with additive noise present) were analysed. The additive noise was placed on the $q, \dot{q}, W, \dot{W}, U$, and $a_{z}$ outputs required by the estimation model outlined in section 2.4. Furthermore, for the Dutch Roll mode noise was also added to $p, r, \dot{r}, V, \dot{V}, U$, and $a_{y}$. A standard Gaussian (zero mean and unit variance) noise was added to the channels, such that the signal to noise ratio $(\mathrm{S} / \mathrm{N})$ was 5 to 1 . The $\mathrm{S} / \mathrm{N}$ was chosen as it provided representative noise, [18] sufficient to corrupt the signals to test the effects of reconstructing and differentiating $\dot{W}$ and $\dot{V}$ on the parameter estimation results. Before performing PE for the conditions with added noise standard practice for flight data analysis was followed by smoothing the data using the Fourier smoothing algorithm developed in [9]. Finally, as a simulation model is being used, the true clean response for the dependent variables $\dot{q}, \dot{W}, \dot{r}$, and $\dot{V}$ can be directly compared with the model predicted response and this enables the two methods to be directly compared.

\begin{tabular}{lccccc}
\hline$\hat{\theta}$ & Linmod & Dif & $s(\hat{\theta})$ & $\operatorname{Rec}$ & $s(\hat{\theta})$ \\
\hline$z_{w}$ & -4.139 & -4.173 & 0.020 & -4.115 & 0.009 \\
$z_{q}$ & 24.33 & 24.44 & 0.091 & 24.27 & 0.027 \\
$z_{\eta}$ & -2.361 & -1.726 & 0.253 & -2.352 & 0.072 \\
$m_{w}$ & -4.289 & -4.420 & 0.005 & -4.427 & 0.008 \\
$m_{q}$ & -6.035 & -6.237 & 0.015 & -6.311 & 0.025 \\
$m_{\eta}$ & -32.54 & -33.27 & 0.038 & -33.30 & 0.064 \\
\hline
\end{tabular}

Table 2: Derivatives and standard errors (Clean)

\begin{tabular}{lccccc}
\hline$\hat{\theta}$ & Linmod & Dif & $s(\hat{\theta})$ & $\operatorname{Rec}$ & $s(\hat{\theta})$ \\
\hline$z_{w}$ & -4.139 & -4.489 & 0.174 & -3.594 & 0.297 \\
$z_{q}$ & 24.33 & 24.17 & 0.599 & 23.80 & 0.622 \\
$z_{\eta}$ & -2.361 & -2.835 & 1.327 & -3.163 & 1.419 \\
$m_{w}$ & -4.289 & -4.844 & 0.250 & -4.296 & 0.389 \\
$m_{q}$ & -6.035 & -2.110 & 0.935 & -3.179 & 1.081 \\
$m_{\eta}$ & -32.54 & -20.58 & 2.315 & -21.44 & 2.462 \\
\hline
\end{tabular}

Table 3: Derivatives and standard errors (Noise)

\begin{tabular}{lccc}
\hline Method & $\omega_{s p}$ & $\omega$ & $\zeta$ \\
\hline Linmod & 11.37 & 10.17 & 0.4473 \\
Differentiated & 11.57 & 10.34 & 0.4495 \\
Differentiated noise & 11.25 & 10.75 & 0.2932 \\
Reconstructed & 11.55 & 10.30 & 0.4513 \\
Reconstructed noise & 10.66 & 10.11 & 0.3176 \\
\hline
\end{tabular}

Table 4: Frequencies (rad/s) and damping ratios 


\subsection{SPPO Results}

In this experiment the Aerosonde model was excited using a $2^{\circ}$ elevator doublet from a SLF trim condition at $48.6 \mathrm{kts}(25 \mathrm{~m} / \mathrm{s})$ and $h$ of $3048 \mathrm{ft}(1000 \mathrm{~m})$. The $W$ and $q$ responses for reconstruction and differentiation (denoted by Rec [dotted line], and Dif [dash-dotted line] respectively) for the clean data are shown alongside the available measured simulation model values (denoted by Sim [solid line]) in figure 3 . The responses from the identified models with signal noise are presented in figure 4 , and the associated state derivative responses $\dot{W}$ and $\dot{q}$ are shown in figure 5. The parameter estimates for the SPPO mode and their standard errors are presented in table 2 for the data without noise and in table 3 for the data with noise. In addition, the parameters from Linmod of the full order system are included in the first column of each table. Finally, the natural frequencies, frequencies of oscillation, and damping ratios for both conditions from reconstruction and differentiation are shown along with the Linmod values in table 4.
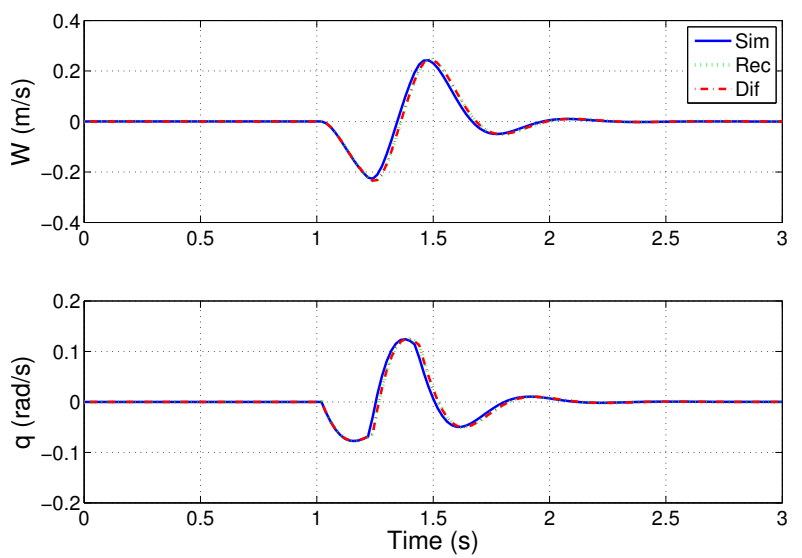

Figure 3: Aerosonde $W$ and $q$ comparison (Clean)
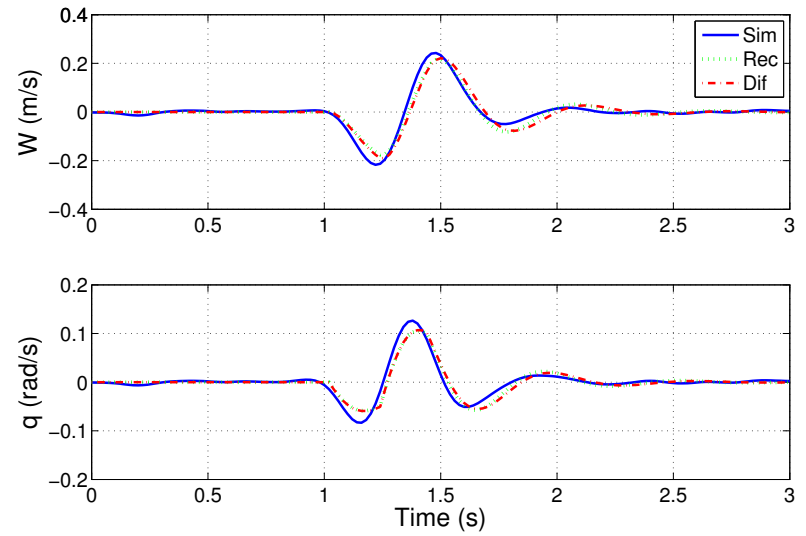

Figure 4: Aerosonde $W$ and $q$ comparison (Noise)
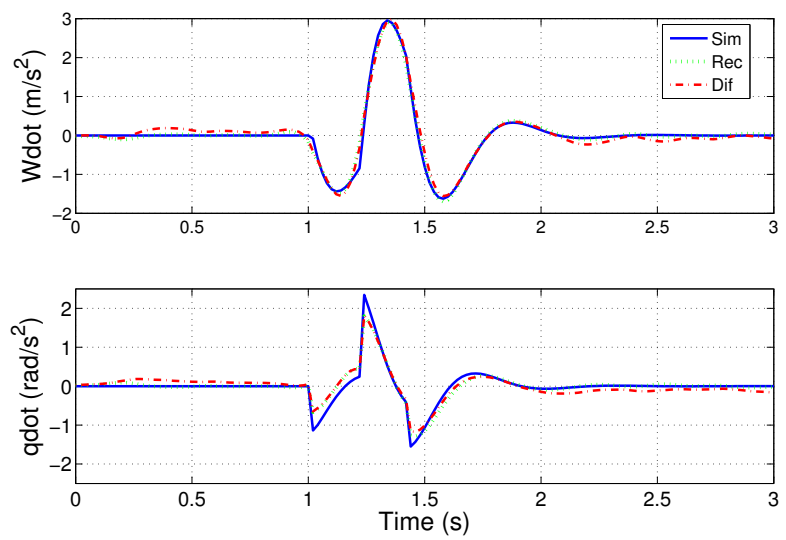

Figure 5: Aerosonde $\dot{W}$ and $\dot{q}$ comparison (Noise)

\subsection{Dutch Roll Results}

The lateral analysis was performed for the same trim condition outlined in section 3.2 , with the $2^{\circ}$ doublet inputs applied to the rudder. Using equation (21) the necessary pulse widths to excite the DR mode were calculated to be 0.3846 seconds. Using the side velocity, $V$ and yaw rate, $r$ responses for reconstruction and differentiation (denoted by Rec [dotted line], and Dif [dashed-dotted line] respectively) for the clean data are shown alongside the available measured simulation model values (denoted by Sim [solid line]), in figure 6 . The predicted $V$ and $r$ model responses for the simulation with noise are shown in figure 7 
and figure 8 shows the associated state derivative responses for $\dot{V}$ and $\dot{r}$.

\begin{tabular}{cccccc}
\hline$\hat{\theta}$ & Linmod & Dif & $s(\hat{\theta})$ & $\operatorname{Rec}$ & $s(\hat{\theta})$ \\
\hline$y_{v}$ & -0.643 & -1.101 & 0.044 & -0.782 & 0.039 \\
$y_{r}$ & -25.02 & -26.37 & 0.252 & -25.96 & 0.218 \\
$y_{\zeta}$ & 3.517 & 4.858 & 1.506 & 4.406 & 1.330 \\
$n_{v}$ & 0.684 & 1.310 & 0.009 & 1.304 & 0.012 \\
$n_{r}$ & -1.043 & -1.399 & 0.068 & -1.712 & 0.089 \\
$n_{\zeta}$ & -22.10 & -21.12 & 0.397 & -20.98 & 0.518 \\
\hline
\end{tabular}

Table 5: Derivatives and standard errors (Clean)

\begin{tabular}{cccccc}
\hline$\hat{\theta}$ & Linmod & Dif & $s(\hat{\theta})$ & $\operatorname{Rec}$ & $s(\hat{\theta})$ \\
\hline$y_{v}$ & -0.643 & -1.036 & 0.094 & -0.752 & 0.041 \\
$y_{r}$ & -25.02 & -26.91 & 0.543 & -26.06 & 0.221 \\
$y_{\zeta}$ & 3.517 & 2.649 & 2.826 & 4.006 & 1.348 \\
$n_{v}$ & 0.684 & 1.282 & 0.022 & 1.273 & 0.028 \\
$n_{r}$ & -1.043 & -1.258 & 0.145 & -1.618 & 0.158 \\
$n_{\zeta}$ & -22.10 & -19.17 & 0.756 & -19.78 & 0.846 \\
\hline
\end{tabular}

Table 6: Derivatives and standard errors (Noise)

The lateral aerodynamic derivatives for the $2 \mathrm{DOF}$ DR model for both conditions are given in tables 5 and 6; again the linearised simulation model DR parameters are presented in the first column of each table. The Dutch roll natural frequencies, frequencies of oscillation, and damping ratios for the respective models are collected in table 7 .

\begin{tabular}{lccc}
\hline Method & $\omega_{d r}$ & $\omega$ & $\zeta$ \\
\hline Linmod & 5.977 & 5.838 & 0.2139 \\
Differentiated & 5.998 & 5.866 & 0.2084 \\
Differentiated noise & 5.984 & 5.873 & 0.1917 \\
Reconstructed & 5.932 & 5.800 & 0.2102 \\
Reconstructed noise & 5.865 & 5.744 & 0.2020 \\
\hline
\end{tabular}

Table 7: Frequencies (rad/s) and damping ratios
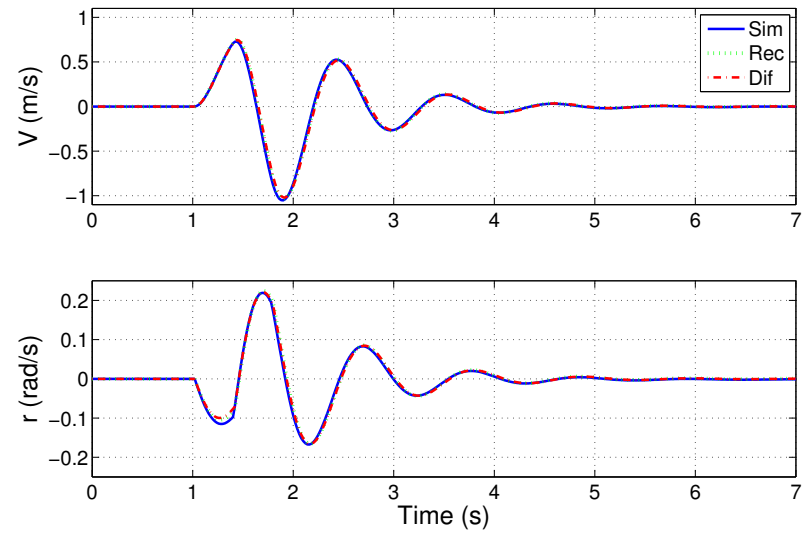

Figure 6: Aerosonde $V$ and $r$ comparison (Clean)
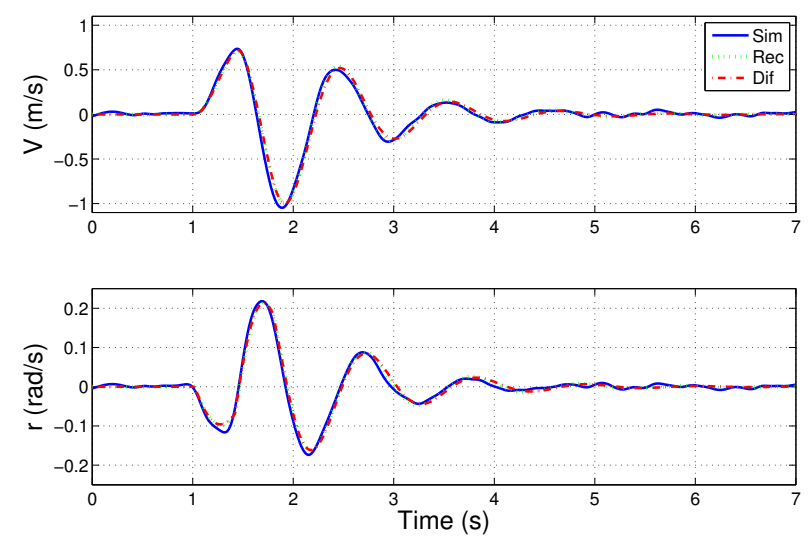

Figure 7: Aerosonde $V$ and $r$ comparison (Noise) 

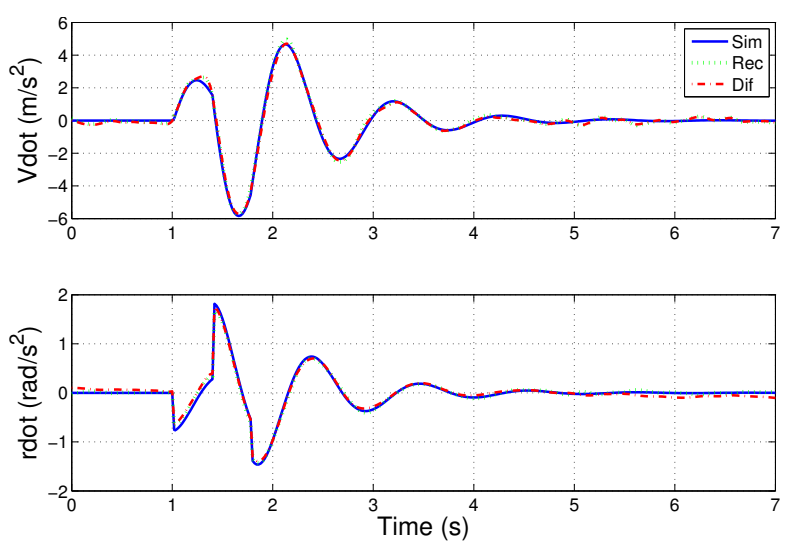

Figure 8: Aerosonde $\dot{V}$ and $\dot{r}$ comparison (Noise)

\section{Flight Test Example}

The BAe Jetstream-31 aircraft was launched in 1978 as a successor to the Handley Page HP-137. Originally designed as a passenger aircraft it has also been deployed in training and maritime defence roles. The aircraft is powered by two Garrett TPE-331-10UG turbo-propellers each capable of producing $701 \mathrm{~kW}$ (940shp), and can carry 18 passengers with baggage and fuel reserves at a cruising speed of $230 \mathrm{kts}$ (at $25,000 \mathrm{ft})$ with a maximum range of $680 \mathrm{~nm}(1260 \mathrm{~km})$ and MTOW 6,950kg.

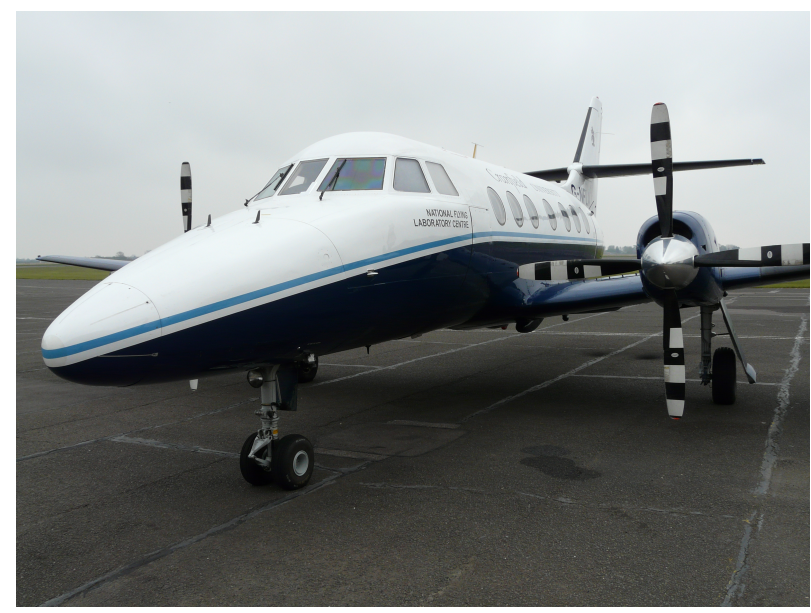

Figure 9: Cranfield's Jetstream-31 G-NFLA

\subsection{Approach}

The primary role of the Cranfield Jetstream aircraft (G-NFLA) is to act as a flying classroom to demonstrate flight dynamics. As a part of such demonstrations, manoeuvres that excite the aircraft's dynamic modes are performed by the crew in accordance with CAA regulations; subsequently this restricts the types of inputs that can be used. The aircraft is equipped with several sensors which enable its dynamic response to be recorded. Flight data for the SPPO mode to test the effects of reconstruction and differentiation of $\dot{W}$ on the parameter estimates were made available. Certain sensors such as the $\alpha$ vane, output analogue signals, and therefore, require digitisation of the data; this introduces an associated time delay (see [19]).

Furthermore, the data onboard the aircraft is smoothed by a hardwired low-pass second order butterworth filter with the cutoff frequency set at $8 \mathrm{~Hz}$; nevertheless, some noise is still present in the recorded signals. To evaluate the identified models we cannot rely on an independent linearised model such as for the Aerosonde UAV since one does not exist. Therefore, the only means to evaluate the models is to compare their predicted time histories for data from a different manoeuvre under the same flight conditions and excitation input. A close match between the predicted output and the validation data time histories is then used to indicate whether the aircraft dynamics have suitably been captured.

\subsection{Jetstream Results}

The results shown are for the following trim condition: $V_{T A S}=170 \mathrm{kts}(87.4 \mathrm{~m} / \mathrm{s}), h=6080 \mathrm{ft}(1853 \mathrm{~m})$ and a c.g. of $23 \%$ MAC. The estimated derivatives are presented in table 8 alongside the standard errors. The validation responses from the estimated reduced order models by the two methods for: the heave $(W)$, and pitch rate $(q)$ are plotted in figure 10, where the reconstruction and differentiation are denoted by Rec (dotted line) and Dif (dash-dotted line), and compared with the measured flight data denoted by Measured-IRS (solid line). The state derivative response plots can be seen in figure 11 and the 
Measured-IRS time history is determined by differentiation of the measured $W$ and $q$ states to yield $\dot{W}$ and $\dot{q}$.

\begin{tabular}{lcccc}
\hline$\hat{\theta}$ & $\operatorname{Dif}$ & $s(\hat{\theta})$ & $\operatorname{Rec}$ & $s(\hat{\theta})$ \\
\hline$z_{w}$ & -0.757 & 0.048 & -1.127 & 0.031 \\
$z_{q}$ & 74.02 & 2.136 & 86.41 & 0.599 \\
$z_{\eta}$ & 2.737 & 3.447 & -6.763 & 1.205 \\
$m_{w}$ & -0.050 & 0.005 & -0.053 & 0.004 \\
$m_{q}$ & -0.971 & 0.154 & -0.695 & 0.153 \\
$m_{\eta}$ & -7.079 & 0.306 & -6.823 & 0.291 \\
\hline
\end{tabular}

Table 8: Derivatives and standard errors
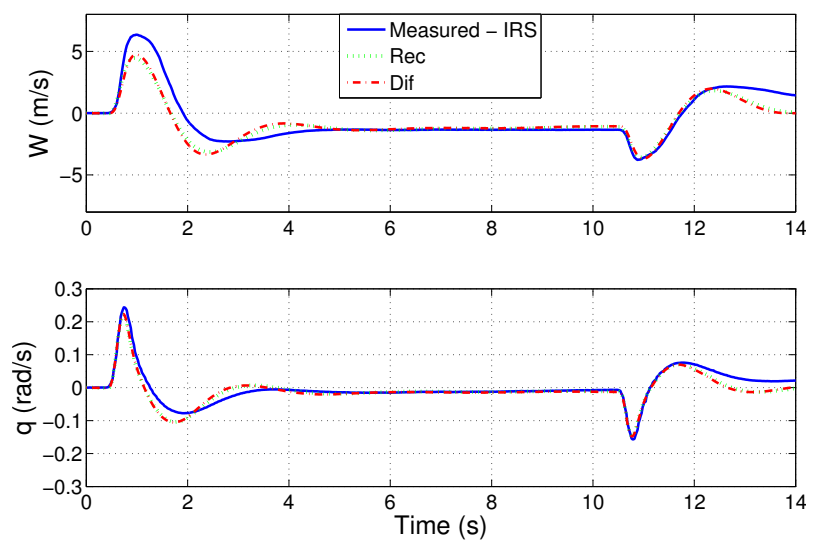

Figure 10: $W$ and $q$ validation results

\section{Discussion}

\subsection{Aerosonde}

SPPO: Referring to the clean data case, both sets of parameter estimates in table 2 agree closely with the linearised model values. In addition the heave and pitch responses in figure 3 show closely fitting matches for both methods, as would be expected for this clean condition. The parameter estimates with a $\mathrm{S} / \mathrm{N}$ of 5 to 1 show a greater variation with respect to the linearised values in table 3 . This results in slight offsets for both methods in the predicted $W$ and $q$ time histories in figure 4. However, the estimated
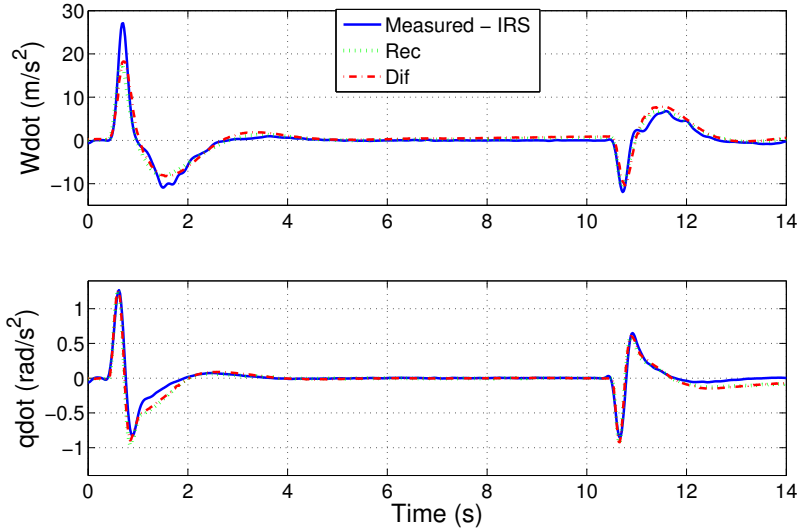

Figure 11: $\dot{W}$ and $\dot{q}$ validation results

state derivative responses with noise, figure 5 , can be seen to follow the clean simulation outputs. In the case with noise the $z_{\eta}, m_{q}$, and $m_{\eta}$ estimated derivatives for both methods have the largest discrepancies from the linearised values, and have the greatest parameter standard errors.

Comparing the linearised natural frequency $\omega_{s p}=$ $11.37 \mathrm{rad} / \mathrm{s}$ with the predicted values in table 4 , the clean data cases predict similar frequencies that are $\sim 0.2 \mathrm{rad} / \mathrm{s}$ higher, and the noise cases predict lower frequencies. In the cases with noise, the differentiated natural frequency is $\omega_{s p}=11.25 \mathrm{rad} / \mathrm{s}$ and the lower reconstruction estimate is $\omega_{s p}=10.66 \mathrm{rad} / \mathrm{s}$. Referring to the respective damping ratios, the results with noise show a reduction in damping ratio, with the differentiation method having the lowest value, $\zeta=0.2932$ compared to $\zeta=0.3176$ for reconstruction. These differences can be explained by observing the principal derivatives that define the SPPO dynamics, $z_{w}, m_{w}$ and $m_{q}$.

With reference to table 3 , the principal difference can be seen in the $z_{w}$ and $m_{q}$ derivatives. For $z_{w}$, the differentiated and reconstructed values are -4.489 and -3.594 respectively, which are close to the linearised value of -4.139. However, in the case of $m_{q}$ the differentiation and reconstruction values are -2.110 and -3.179 , respectively, which are both significantly higher than the linearised value of -6.035 . Therefore, the high $m_{q}$ estimate can be seen as the culprit responsible for the lower estimated natural frequencies 
and damping ratios due to the resultant products and sums between the respective $m_{q}$ and $z_{w}$ values. Finally, it is important to highlight that the $2 \mathrm{DOF}$ parameter estimates are being compared with the 6 DOF linearised model values. As a result the latter will contain more information and leads to such differences in the parameter estimates. Overall the reconstructed $\dot{W}$ data was able to provide similar reliable results to those determined with the differentiated $\dot{W}$.

$D R$ : Observing the side velocity and yaw rate responses in figures 6 and 7 ; following the excitation the predicted response for both methods matches closely with the simulation output. The presence of signal noise can be seen to slightly offset the predicted responses in figure 7 ; the state derivative responses in figure 8 follow the general trend of the clean simulation data. The estimated parameters with no noise, in table 5, show good agreement between both methods and have low standard errors. In the presence of signal noise the majority of derivatives matched the linearised values well. Comparing the parameter estimates in tables 5 and 6 it can be seen that the reconstructed estimates have mostly lower standard errors. The largest discrepancies occur for the side velocity terms $y_{v}$ and $n_{v}$, with both approximately $50 \%$ of the linearised values; despite this, the predicted responses matched well. A possible explanation for this occurring could be due to the Ordinary least squares fit, which simply returns the best parameters which minimise the equation error without applying any weighting on the regressors.

The predicted natural frequencies in table 7 showed good agreement, with the reconstructed method's results being slightly lower. Nevertheless, the associated damping ratios for the reconstructed method matched the linearised values closer in both the clean and noise cases. Primarily, the yawing moments $\left(n_{v}\right.$, $n_{r}$, and $\left.n_{\zeta}\right)$ will have some contributions due to roll which in the present model (see equation (9) in section 2.4) are not accounted for due to the $2 \mathrm{DOF}$ model. Furthermore, in the $\dot{V}$ reconstruction equation (equation (15)) the effects due to roll were also eliminated, and therefore, could be responsible for the differences in $y_{v}$ and $n_{v}$, and the difficulty in capturing the DR mode $n_{v}$ when signal noise is added.
As an aside while undertaking this research the authors found that the choice of input to excite the model had a significant effect on the predicted time history fit. Subsequently, this led to the selection of elevator and rudder doublet inputs targeted to excite the target bandwidth of the Aerosonde closest to its natural frequency which minimised the offset and respected the small perturbation assumption.

\subsection{Jetstream}

The evaluation of the Jetstream 2 DOF SPPO models can only be validated by using the estimated model parameters from the test case and observing their predicted responses for a different set of data. The difference between the results from the two techniques are difficult to discern as highlighted by the responses in figures 10 and 11 with both techniques showing good tracking of the measured response.

Analysing the short period parameter estimates in table 8 the $z_{q}, z_{\eta}$ and $m_{\eta}$ derivatives have the largest standard errors, however, the respective standard errors for the reconstructed case were lower. As a rule of thumb the $z_{q}$ estimate should approximately equal $V_{T A S}$, which in this test case was $87.4 \mathrm{~m} / \mathrm{s}$, with the reconstructed estimate providing the closest match. It should also be highlighted that the signs of $z_{\eta}$ do not agree, as the estimate for the differentiated method is positive and has the overall highest standard error for a parameter. Nevertheless, the $m_{q}$ derivatives are both negative; this derivative accounts for the dynamics in the SPPO model as it describes the pitch damping of the oscillatory mode. Previous work [20] has highlighted the sensitivity in identifying the correct sign of $m_{q}$ due to the downwash lag effects from the wing on the tail plane.

The results for the flight test example agree with the SPPO simulation results: that the reconstruction technique can be used to determine the "unmeasurable" $\dot{W}$. Nevertheless, the downside to depending solely on the inertial system in practice is that such a system will be adversely impacted by the presence of external atmospheric disturbances. As a result the reconstruction method is better suited for use on short data recordings, such as those associated with a small perturbation about trim as opposed to replacing air 
data systems for routine use. Therefore, reconstruction provides an alternative means to evaluate the unmeasurable terms $\dot{W}$ and $\dot{V}$ for use in UAV parameter estimation should air data not be available.

\section{Conclusions \& Future Work}

Following this investigation it was found that the reconstruction of $\dot{W}$ and $\dot{V}$ provided reliable results for the SPPO and DR modes respectively. The ability to capture the main underlying dynamics with reduced order models was demonstrated for the case of a simulated UAV and the longitudinal SPPO mode for the Jetstream-31 Laboratory Aircraft (G-NFLA).

Leading on from the present work the opportunity exists to further investigate using the reconstruction method and reduced order a priori models, to perform online UAV parameter estimation. Considering research into parameter estimation for UAVs, the ability to use 2 DOF models will be of great benefit as it reduces the computational complexity of the calculations. Future work will need to address the onboard UAV data processing requirements, in addition to the necessary modifications for the estimation algorithm for online identification. Furthermore, an investigation into quantifying the effects of sensor noise and atmospheric turbulence on the reliability of UAV parameter estimation results using currently available test instrumentation would also be a worthwhile contribution.

\section{Acknowledgements}

This research was funded by a CASE award supported jointly by EPSRC and BAE Systems. The lead author would like to thank Dr. Stephen Carnduff and Dr. Eugene Morelli for their time and advice.

\section{References}

[1] K. W. Iliff. "Parameter estimation for flight vehicles". Journal of Guidance, Control, and Dynamics, Vol 12:pp 609-622, September - October 1989.
[2] P. G. Hamel and R. Jategaonkar. "Evolution of flight vehicle system identification". Journal of Aircraft, Vol 33 (1):pp 9-28, January - February 1996.

[3] S. D. Carnduff. "System Identification of Unmanned Aerial Vehicles". PhD thesis, Cranfield University, Cranfield, Bedfordshire, 2008.

[4] Photo Aerosonde, Atlantic 1998, Wallpaper free for common use, taken from Aerosonde Pty Ltd. www.aerosonde.com, 11/04/2005.

[5] L. A. Zadeh. "From circuit theory to system theory". Proceedings of the IRE, Vol 50:pp 856 - 865, May 1962.

[6] V. Klein. "Estimation of aircraft aerodynamic parameters from flight data". Progress in Aerospace Sciences, Vol 26 (1):pp 1-77, 1989.

[7] V. Klein. "A review of system identification methods applied to aircraft". Technical Report Joint Institute for Acoustics and Flight Sciences Report: N83 33901, The George Washington University, 1983.

[8] M. V. Cook. "Flight Dynamic Principles: A linear systems approach to aircraft stability and control". Elsevier, Amsterdam, 2007.

[9] V. Klein and E. A. Morelli. "Aircraft System Identification: Theory and Practice". AIAA, Reston, Virginia, 2006.

[10] G. Chowdhary, W M. DeBusk, and E. N. Johnson. "Real-time system identification of a small multi-engine aircraft with structural damage". In AIAA Infotec@Aerospace, Atlanta, Georgia, 20th-22nd April 2010. AIAA-2010-3472.

[11] R. Jategaonkar. "Flight Vehicle System Identification: A Time Domain Methodology". AIAA, Reston, Virginia, 2006.

[12] E. A. Morelli. "Practical aspects of the equationerror method for aircraft parameter estimation". In AIAA Atmospheric Flight Mechanics Conference and Exhibit, Keystone, Colorado, 21st-24th August 2006. AIAA-2006-6144. 
[13] C. Lanczos. "Applied Analysis". Sir Isaac Pitman \& Sons, London, 1957.

[14] B. Etkin. Dynamics of Atmospheric Flight. John Wiley \& Sons, 1972.

[15] J. A. Mulder. "Design and Evaluation of Dynamic Flight Test Manoeuvres". PhD thesis, Delft University of Technology, Delft, 1986.

[16] J. F. Whidborne. "A note on the Aerosonde model". Cranfield University, (internal report) 1st edition, July 2007.

[17] G. W. Foster. "The Identification of Aircraft Stability and Control Parameters in Turbulence". PhD thesis, Cranfield Institute of Technology, Cranfield, Bedfordshire, 1982.

[18] E. A. Morelli and M. S. Smith. Real-time dynamic modeling: data information requirements and flight test results. Journal of Aircraft, 46 (6):pp 1894-1905, November-December 2009.

[19] P-D. Jameson. Post-Manoeuvre and Online Parameter Estimation for Manned and Unmanned Aircraft. PhD thesis, Cranfield University, Cranfield, Bedfordshire, 2013.

[20] G. J. Mullen. Aircraft Parameter Identification Using MATLAB. Technical Report College of Aeronautics Report: 0011, Cranfield University, 2000. 


\title{
Cranfield University
}

\section{CERES https://dspace.lib.cranfield.ac.uk}

2015-08-01

\section{Reduced order system identification for UAVs}

\author{
Pierre-Daniel, Jameson
}

Royal Aeronautical Society

P-D. Jameson and A. K. Cooke. Reduced order system identification for UAVs. The

Aeronautical Journal, August 2015, Volume 119, No 1218. pp961-980

http://aerosociety.com/News/Publications/Aero-Journal/Online/3382/Reduced-order-system-identification-for-UAVs Downloaded from Cranfield Library Services E-Repository 